\title{
Visual experimentation
}

\section{Ravindra Athale, Joseph van der Gracht}

Ravindra A. Athale, Joseph van der Gracht, "Visual experimentation," Proc. SPIE 9663, Eighth International Topical Meeting on Education and Training in Optics and Photonics, 96630B (6 October 2003); doi: 10.1117/12.2208376

SPIE Event: Eighth International Topical Meeting on Education and Training in Optics and Photonics, 2003, Tucson, Arizona, United States 


\title{
Visual experimentation
}

\author{
Ravindra A. Athale \\ ECE Department, George Mason University, Fairfax, VA USA \\ telephone: (703) 696-2237; fax:(703)696-2206; e-mail: rathale@darpa.mil
}

\author{
Joseph van der Gracht \\ HoloSpex, Inc., Columbia, MD, USA
}

\begin{abstract}
Very simple visual aids can be designed to convey sophisticated concepts in optics to students ranging from $5^{\text {th }}$ grade to first year graduate students. In this talk I will outline several specific classroom experiments illustrating concepts in wave optics that can be performed with computer generated holograms.

(C)2003 Optical Society of America

OCIS codes: (000.2060) Education
\end{abstract}

\section{Summary}

Difficult concepts are explained much more readily if the instructor can excite students' imagination and capture their attention by providing hands-on manipulation. Concepts in wave optics are most readily amenable to experiments that involve visual effects. Simple diffraction gratings have been used to create a simple spectrometer, which can then be used to analyze the spectra of common light sources. In this talk, we will describe the use of far field computer generated holograms to create interesting visual effects, which can then be used to explore various ideas in wave optics. Experiments can be designed to be easily performed in a classroom setting without elaborate and expensive equipment. The experiments possible with far field computer generated holograms can be both qualitative and quantitative. The sophistication of the experiments can be scaled to be appropriate for students from $5^{\text {th }}$ grade all the way to first year graduate students in optics.

The primary principle of all the experiments to be performed is the interaction between the light originating from the various objects in the scene (self-luminous as well as reflective) and the intervening medium or structures together creating the visual sensation for the observer. The most trivial example of this principle is looking at a scene through colored filters. The effect we will be exploiting in our work is the spatially structured filters that are placed in between the scene and the observer. Again, the most familiar example of such a phenomenon is when one looks at streetlights through a fine mesh screen. Computer generated holograms can be considered to be a vastly more sophisticated and controllable screen which lead to many startling and somewhat counter-intuitive effects. These effects are used as a basis to explain diffraction and other aspects of wave phenomenon. The talk will contain several specifically formulated experiments designed for students at varying level of scientific preparation. 